Patrick Collier, MD, PhD,

FASE, FESC, FACC

Associate Director of the Echo Lab and Co-Director

Cardio-oncology Center; Robert and Suzanne Tom-

sich Department of Cardiovascular Medicine, Sydell

and Arnold Miller Family Heart and Vascular Institute,

Cleveland Clinic; Associate Professor of Medicine,

Cleveland Clinic Lerner College of Medicine of Case

Western Reserve University, Cleveland, $\mathrm{OH}$
Muzna Hussain, MD

Cardiology Research Fellow, Robert

and Suzanne Tomsich Department of

Cardiovascular Medicine, Sydell and Arnold

Miller Family Heart and Vascular Institute,

Cleveland Clinic
Zoran B. Popovic, MD, PhD

Robert and Suzanne Tomsich Department of

Cardiovascular Medicine, Sydell and Arnold

Miller Family Heart and Vascular Institute,

Cleveland Clinic: Associate Professor of

Medicine, Cleveland Clinic Lerner College of

Medicine of Case Western Reserve University,

Cleveland, $\mathrm{OH}$
Brian P. Griffin, MD

Section Head, Imaging, Department of Cardiovascular Imaging, Robert and Suzanne Tomsich Department of Cardiovascular Medicine, Sydell and Arnold Miller Family Heart and Vascular Institute, Cleveland Clinic

\title{
Cardiac surveillance for anti-HER2 chemotherapy
}

\section{ABSTRACT}

Surveillance of left ventricular function, part of current US Food and Drug Administration recommendations for antihuman epidermal growth factor receptor 2 (anti-HER2) chemotherapy, is based on historical data involving patients who received concomitant anthracycline therapy, a key enhancer of cardiac risk. More recent anti-HER2 treatment data suggest that cardiotoxicity detected by screening is rare and usually benign for patients who do not have cardiovascular risk factors and are not taking an anthracycline. Because of the burden of repetitive echocardiography required for surveillance and the risk of false-positive results, potentially leading to discontinuing lifesaving treatment, we advocate for a more focused cardiac surveillance strategy.

\section{KEY POINTS}

Accurate diagnosis of cardiotoxicity is critical, as falsepositive results may lead to inappropriate stopping of potentially lifesaving chemotherapy.

We suggest routine serial measurement of left ventricular ejection fraction by echocardiography only for patients who have received anthracyclines or are considered at high cardiac risk.

\section{All patients should be counseled to promptly report relevant symptoms.}

For patients who develop clinically significant congestive heart failure, discontinuing anti-HER2 therapy should be strongly considered.
A NTI-HUMAN EPIDERMAL GROWTH FACTOR receptor 2 (anti-HER2) therapy has been a game-changer for some forms of aggressive breast cancer, drastically reducing mortality rates. The US Food and Drug Administration (FDA) calls for close cardiac surveillance in patients receiving these drugs. But this recommendation is based on an early clinical trial with circumstances that are no longer frequently relevant. As it now stands, this strategy is burdensome, dangerous for patients who are unnecessarily advised to discontinue therapy, and often ignored in practice.

This article discusses what drove the current FDA cardiac surveillance strategy for anti-HER2 therapy and the challenges it poses in practice and clinical research. Results of more recent clinical trials are reviewed, and in light of them, new best practices for anti-HER2 therapy management and cardiac monitoring are proposed.

\section{HER2 EFFECTS, TESTING, AND THERAPY}

About 1 in 4 patients with breast cancer has an aggressive tumor that overexpresses a tyrosine kinase receptor protein called human epidermal growth factor receptor 2 (HER2, also known as HER2/neu, CD340, Erbb2, and proto-oncogene $\mathrm{Neu}$ ). It is encoded by the ERBB2 oncogene on chromosome 17. Signaling through this receptor promotes cell proliferation and opposes apoptosis; when it is overexpressed, uncontrolled cell growth results.

Patients with breast cancer undergo HER2 testing to assess prognosis and determine candidacy for personalized therapy. Over the past 20 years, agents targeted against HER2 have been developed, contributing to a halving of 
TABLE 1

\section{Trials involving anti-HER2 treatment}

\begin{tabular}{|c|c|c|c|c|c|c|c|c|c|c|}
\hline Study $^{\mathrm{a}}$ & Year & $\begin{array}{l}\text { No. of } \\
\text { patients }\end{array}$ & $\begin{array}{l}\text { Duration } \\
\text { (years) }\end{array}$ & $\begin{array}{l}\text { Early vs } \\
\text { metastatic }\end{array}$ & $\begin{array}{l}\text { Anthra- } \\
\text { cycline }\end{array}$ & $\begin{array}{l}\text { Follow-up } \\
\text { (years) }\end{array}$ & $\begin{array}{l}\text { Echo- } \\
\text { cardiog- } \\
\text { raphy } \\
\text { result }\end{array}$ & $\begin{array}{l}\text { LVEF } \\
\text { drop } \\
(\%)\end{array}$ & $\begin{array}{l}\text { Heart } \\
\text { failure } \\
\text { incidence } \\
(\%)\end{array}$ & $\begin{array}{l}\text { Cardiac } \\
\text { death } \\
(\%) \\
\end{array}$ \\
\hline OHERA $^{7}$ & 2019 & 3,733 & 1 & Early & Yes & 5 & $4+$ & 2 & 3 & $<1$ \\
\hline KATHERINE $^{8}$ & 2019 & 1,486 & 0.4 & Early & No & 5 & $4+$ & NA & NA & 0 \\
\hline NSABP9 & 2017 & 407 & 1 & Early & Yes & 5 & $4+$ & 1 & NA & $<1$ \\
\hline HERA $^{10}$ & 2017 & 5,099 & $1-2$ & Early & No & 10 & $4+$ & $<1$ & NA & NA \\
\hline APHINITY $^{11}$ & 2017 & 4,805 & 1 & Early & No & 10 & $5+$ & NA & $<1$ & $<1$ \\
\hline Dang et al ${ }^{12}$ & 2016 & 406 & 1 & Both & No & 4 & $4+$ & 3 & $<1$ & NA \\
\hline $\mathrm{HORG}^{13}$ & 2015 & 481 & 1 & Early & No & 7 & $3+$ & $<1$ & 0 & 0 \\
\hline CLEOPATRA $^{14}$ & 2013 & 804 & 1 & Both & No & 3 & $3+$ & 1 & $<1$ & $<1$ \\
\hline NeoSphere $^{15}$ & 2012 & 417 & 0.4 & Both & No & 2 weeks & $3+$ & $<1$ & $<1$ & 0 \\
\hline BCIRG-006 ${ }^{16}$ & 2011 & 3,222 & 1 & Both & Yes & 5 & $7+$ & 14 & $<1$ & 0 \\
\hline Slamon et al & 2001 & 234 & 0.8 & Metastatic & Yes & $>2$ & NA & 16 & 27 & 0 \\
\hline \multicolumn{11}{|c|}{$\begin{array}{l}\text { aAll trials included radiation therapy and were adjudicated. } \\
\text { bPivotal trial leading to stringent US Food and Drug Administration recommendations for cardiac surveillance. } \\
\text { APHINITY = A Study of Pertuzumab in Addition to Chemotherapy and Trastuzumab as Adjuvant Therapy in Participants With Human Epidermal Growth Receptor } \\
2 \text { (HER2)-Positive Primary Breast Cancer; BCIRG = Breast Cancer International Research Group; CLEOPATRA = A Study to Evaluate Pertuzumab + Trastuzumab } \\
\text { + Docetaxel vs. Placebo + Trastuzumab + Docetaxel in Previously Untreated HER2-Positive Metastatic Breast Cancer; HER2 = human epidermal growth factor } \\
\text { receptor 2; HERA = HERceptin Adjuvant; HORG = Hellenic Oncology Research Group; KATHERINE = A Study of Trastuzumab Emtansine Versus Trastuzumab as } \\
\text { Adjuvant Therapy in Patients With HER2-Positive Breast Cancer Who Have Residual Tumor in the Breast or Axillary Lymph Nodes Following Preoperative Therapy; } \\
\text { LVEF = left ventricular ejection fraction; NA = not available; NeoSphere = A Study of Pertuzumab in Combination With Herceptin in Patients With HER2 Positive } \\
\text { Breast Cancer; NSABP = National Surgical Adjuvant Breast and Bowel Project; OHERA = Observational Study of Cardiac Events in Patients with HER2-Positive } \\
\text { EBC Treated with Herceptin }\end{array}$} \\
\hline
\end{tabular}

breast cancer mortality rates, in what is widely regarded as a phenomenal success story. ${ }^{2}$ AntiHER2 agents include the following ${ }^{3,4}$ :

Monoclonal antibodies, eg, trastuzumab, which targets the extracellular domain of HER2, and pertuzumab, which prevents HER2 receptor homodimerization and heterodimerization, which are necessary for activation

Ado-trastuzumab emtansine, an antibody-drug conjugate

Small-molecule inhibitors that block the HER2 receptor intracellularly, eg, lapatinib, available in oral formulations.

Other targeted anti-HER2 therapies are continually being developed.

\section{CARDIAC ISSUES WITH TRASTUZUMAB DISCOVERED EARLY}

Current FDA recommendations regarding the frequency of surveillance of left ventricular function with anti-HER2 therapy are conservatively based on historical data involving patients receiving concomitant anthracycline therapy. ${ }^{5}$

The pivotal trastuzumab randomized controlled trial in patients with metastatic breast cancer, published in 2001, reported a 27\% rate of cardiac dysfunction and a 16\% rate of New York Heart Association (NYHA) class III or IV heart failure in patients who received trastuzumab with anthracycline chemotherapy. ${ }^{6}$ These findings prompted the FDA to issue a stern package-insert warning of cardiomyopathy for anti-HER2 treatments, and recommendations for cardiac surveillance.

Trastuzumab's package insert recommends measuring left ventricular ejection fraction (LVEF) before starting therapy, every 3 months during treatment, and at the completion of therapy. If drug therapy is withheld for cardiotoxicity, studies should be repeated 
monthly. Furthermore, after completion of therapy, LVEF should be measured every 6 months for at least 2 years. ${ }^{5}$ Thus, a minimum of 9 echocardiograms is recommended for patients undergoing a standard 12 -month adjuvant dosing schedule, with an indefinite (and potentially lifelong) number of 3-monthly echocardiograms for those with metastatic disease on continual anti-HER2 therapy.

\section{RECENT DATA PUT RECOMMENDATIONS IN QUESTION}

Subsequent clinical trials ${ }^{7-16}$ have generally indicated a more favorable cardiac profile (Table 1 ).

The 2007 Herceptin Adjuvant (HERA) trial found a $3 \%$ rate of cardiac dysfunction and a $0.6 \%$ rate of NYHA III or IV heart failure. ${ }^{17}$ A 2019 trial $^{8}$ found that only $1.2 \%$ of patients discontinued dual therapy because of decreased ejection fraction, while adjudicated cardiac events occurred in less than $1 \%$. The relationship between decreased ejection fraction assessed by cardiac monitoring and the development of clinical heart failure was not discussed.

Although the risk of cardiac dysfunction

Current

recommen-

dations are

conservatively

based on historical data from anti-HER2 therapy now appears low, the FDA package-insert warning and recommendations remain. Extensive cardiac monitoring and echocardiographic testing regimens are still part of the standard protocols of clinical trials involving this drug., ${ }^{8,11,18,19}$ In a 2017 trial, ${ }^{11}$ up to 13 imaging studies (preferably echocardiograms) were scheduled using the following protocol: at baseline, during treatment (at chemotherapy cycles 2, 6, 10, and 14) and during follow-up (months 3, 6, 12, 18, $24,36,48,60)$, resulting in a potential total of 19,318 studies for 1,486 patients.

\section{WHAT ACCOUNTS FOR DIFFERENT RESULTS BETWEEN TRIALS?}

Several factors may help explain different event rates between clinical trials of the same drug.

Concomitant vs sequential therapy. In early studies, trastuzumab was given concomitantly with an anthracycline and cyclophosphamide. It has since been realized that cardiotoxicity rates are much lower if trastuzumab is given sequentially with other drugs. This is likely the most important explanation of the differences between the early and late anti-HER2 clinical trials.

More surveillance in the drug arm. A 2019 long-term study ${ }^{20}$ found a higher rate of cardiotoxicity in patients treated with trastuzumab than in those treated with chemotherapy alone. But LVEF was measured 5 times in the trastuzumab group vs no routine testing in the control group. Because cardiotoxicity is more likely to be revealed if more LVEF measurements are taken, more surveillance usually results in findings in the more tested arm.

Exclusion criteria. Cardiac event rates may be underestimated in clinical trials that exclude high-risk patients who are more likely to experience such events.

Problems of definition. Duplicative, inconsistent, and sometimes contradictory consensus criteria to classify cardiotoxicity can affect event rates. For example, a study participant experiencing an asymptomatic drop in LVEF from $60 \%$ to $35 \%$ might be reported as having either grade 0 left ventricular dysfunction, grade 1 heart failure, or a grade 3 ejection fraction decrease. ${ }^{21,22}$

Method of event reporting. Variability in reported outcomes data can arise if studies only include adverse events that are "site reported." 23 But this is less relevant for objective findings, such as drop in ejection fraction, which should be documented in the primary data. Ideally, all events, whether or not they are thought to be treatment-related, are reported, with details provided for events that are believed not to be treatment-related. ${ }^{23}$

Findings from screening using surveillance echocardiography would probably not be confused with acute events associated with other temporary or persisting causes of left ventricular dysfunction (eg, sepsis, acute coronary syndrome, acute arrhythmia including atrial fibrillation, takotsubo cardiomyopathy).

\section{PRINCIPLES OF CARDIAC SURVEILLANCE}

More than 50 years ago, Wilson ${ }^{24}$ wrote about the attributes of an ideal screening test and advised caution: "In theory, screening is admirable, but in practice there are snags; the central idea is simple and may appear deceptively straightforward." Wilson's screening criteria and their applications to surveillance echo- 
TABLE 2

\section{Wilson's criteria for an ideal screening test, applied to cardiac surveillance for chemotherapy}

Criteria ${ }^{24}$

The natural history of the condition should be understood

There should be a recognizable latent or early symptomatic stage

A test should exist that is easy to perform and interpret, and is acceptable, accurate, reliable, sensitive, and specific

An accepted treatment for the disease should exist

Treatment should be more effective if started early

There should be a policy on who should be treated

Diagnosis and treatment should be cost-effective

Case-finding should be a continuous process

cardiography during chemotherapy are presented in Table $2 .{ }^{24}$

\section{LVEF WITH ECHOCARDIOGRAPHY IS RECOMMENDED FOR SCREENING}

Currently, LVEF is the screening variable of choice. ${ }^{19}$ Strain assessment is a nonactionable supportive tool. However, it is the focus of ongoing research and is increasingly being used, especially as it received a formal Current Procedural Terminology code by the US Centers for Medicare and Medicaid for reimbursement to Medicare providers. ${ }^{25}$

Echocardiography is the preferred screening method, although cardiac magnetic resonance imaging is considered to be the gold
Surveillance echocardiography for chemotherapy

Cardiotoxicity is an important health problem but is detectable by screening only in a minority of patients

The natural history of cardiotoxicity has been reasonably well studied for established chemotherapy agents such as anti-HER2

Left ventricular dysfunction typically relates to acute toxicity and becomes manifest within the first year of exposure. Early recognition is important, because cumulative doses typically compound toxicity

Imaging with echocardiography has these qualities but also involves considerable challenges and limitations

Current guideline-directed heart failure management is recognized as treatment for chemotherapy-related cardiomyopathy. Evidence is limited for specific treatments beyond these guidelines, although the subject is under active investigation

If started early, current guideline-directed heart failure management is considered to be more effective. Early recognition of chemotherapyrelated cardiomyopathy is important for preventing additional dose exposures, which typically compound toxicity

Current guideline-directed heart failure management covers who should be treated

Limited data suggest favorable cost-effectiveness for screening and early treatment, although a more targeted approach can likely significantly improve it

Case-finding can be a continuous process

standard and is advised in selected cases (ie, if echocardiographic images are inadequate or yield equivocal findings). Another option, multigated acquisition radionuclide scanning, is not a first-line test, as it involves radiation and introduces cross-modality error.

\section{BALANCING THERAPY RISKS AND BENEFITS}

The net benefit of therapy refers to balancing the risks of toxicity with prognosis and available treatment options. Potential cardiotoxicity may be more acceptable in the setting of a cancer with a poor prognosis and few treatment possibilities. On the other hand, cardiotoxicity is less likely to be an acceptable risk 
for a later-generation drug in a cancer with multiple existing therapies and a generally good prognosis.

Regarding breast cancer, regimens without an anthracycline have been shown to be as effective as those with an anthracycline, especially for women at low risk of recurrence. Strategies without an anthracycline involve much lower rates of cardiotoxicity, with rates of NYHA class III and IV heart failure being close, if not equal, to those with placebo $(0.4 \%$ over 5 years or fewer than 1 per 1,000 patients per year). ${ }^{18}$ They have also demonstrated improved survival and favorable cardiac safety for metastatic cancer. ${ }^{26}$

Because anti-HER2 treatment is used against a particularly aggressive cancer, decisions regarding interrupting or stopping it based on side effects have especially important implications. Whether such decisions should be made based on a surrogate echocardiographic end point, possibly in the absence of symptoms, needs careful consideration.

\section{CARDIAC MANAGEMENT AND ANTI-HER2 THERAPY}

\section{Echocardio- graphy is the preferred screening method, although MRI is considered the gold standard}

Anti-HER2 treatment in patients with preexisting cardiac dysfunction has been associated with a worse prognosis and higher rate of symptomatic heart failure compared with patients with preserved ejection fraction at baseline. ${ }^{27}$ However, preexisting cardiac dysfunction is a relative rather than an absolute contraindication to starting anti-HER2 treatment. The FDA recommends extreme caution in treating such patients, ${ }^{5}$ and a cardiologist should be involved in management.

For patients who develop clinically significant congestive heart failure, discontinuing anti-HER2 therapy should be strongly considered. For patients without symptoms, treatment-specific LVEF thresholds for stopping medications have been developed, with slightly different recommendations between FDA-approved labeling, clinical trial protocols, and professional society guidelines. Criteria from clinical trials that do not involve anthracycline therapy tend to be a little less stringent because anti-HER2associated toxicity is considered to be doseindependent, nonapoptotic, and potentially reversible (type 2 cardiotoxicity). In contrast, anthracycline-mediated cardiotoxicity is regarded as type 1 (ie, irreversible and related to cumulative dose). ${ }^{28}$

A commonly used threshold defining cardiotoxicity is a decrease in LVEF of more than $10 \%$ to a value below the lower limit of normal. Hussain et $\mathrm{al}^{29}$ in a study of $23 \mathrm{pa}$ tients with asymptomatic LVEF decline who continued trastuzumab, found that 14 patients $(61 \%)$ tolerated it without a cardiac event, 6 (26\%) developed further worsening of LVEF, 1 (4\%) developed heart failure, and 2 (9\%) died of a possible or probable cardiovascular cause.

Strategies to prevent or attenuate cardiotoxicities include participation in cardio-oncology programs (particularly for symptomatic or high-risk patients being considered for antiHER 2 treatment, including anyone with baseline low LVEF), early recognition of cardiac side effects, active cardiac surveillance, and cardioprotective medical therapy.

\section{INTERPRETING SERIAL TESTING IS A CHALLENGE}

Accurate diagnosis of cardiotoxicity is critical, as false-positive results may lead to inappropriate stopping of potentially lifesaving chemotherapy.

Serial echocardiography in patients with cancer can be difficult and measurement variability may be high. Reasons may be technical (eg, concomitant lung disease, high or low body mass, postoperative status) or involve confounding factors (eg, variable hemodynamics, medications, fluid status).$^{30}$ Published test-retest variability data have generally been derived between 2 tests rather than multiple tests and conducted under optimized experimental settings in academic centers. Even optimized test-retest variability remains close to echocardiographic thresholds used to define real interval change representative of true cardiotoxicity, especially with multiple tests.

Outside of trial settings, false-positive results are not infrequent. In addition, prechemotherapy studies may manifest hyperdynamic function. Teasing out whether serial changes are related to cancer therapy vs comorbid illness (eg, concomitant arrhythmia, ischemia, stress cardiomyopathy, and myocarditis) may be challenging. 


\section{Optimizing measurement accuracy}

Repeat echocardiographic studies should be performed in as consistent a manner as possible (eg, same equipment, technician, and reporting physician). Multiple ways to measure LVEF should be used, including quality 3-D echocardiography (ideally without contrast for highest reproducibility), the biplane Simpson method (with contrast, if necessary), and visual assessment, with reporting of the best available data. ${ }^{19}$ Global longitudinal strain may provide corroborative data when concordant; if discordant, the quality of the data should be reviewed again with particular attention to wall tracking. Ideally, differences between serial tests should be compared to the maximal detectable difference, a value that can be calculated for each echocardiographic laboratory, providing a threshold to distinguish likely test error from real change. ${ }^{31}$ For borderline cases, obtaining an experienced second opinion, an early interval repeat study, or an alternative test (eg, cardiac magnetic resonance imaging) should be considered.

\section{Treating chemotherapy-related cardiomyopathy remains a challenge}

Applying current guideline-directed heart failure management to chemotherapy-related cardiomyopathy can be particularly challenging. Especially for patients undergoing chemotherapy, titrating levels of beta-blockers and renin-angiotensin system antagonists to optimal dosing is often difficult because of poor tolerability. Outside these guidelines, data to support the use of cardioprotective medical therapy to prevent chemotherapy-related cardiotoxicity are modest at best. Studies are limited by marginal effect size, small patient numbers, and short follow-up.

\section{Should general cardiotoxicity screening be eliminated?}

Many have questioned the usefulness of currently proposed cardiac monitoring for patients on anti-HER2 therapy, particularly for those who are asymptomatic, without cardiovascular risk factors, and who have not had concomitant anthracycline therapy. ${ }^{32}$ Data assessing the cost-effectiveness of screening strategies for cardiotoxicity are limited..$^{33}$ To avoid placing additional financial and time burdens on patients with cancer and their families, some have suggested that simply monitoring patients on clinical parameters alone is best. ${ }^{26}$

Current practice regarding screening for chemotherapy-related cardiomyopathy is a legacy of its mutable historical background. It is overshadowed by variable and conflicting guidelines, with the result that most patients on anti-HER2 treatment actually receive minimal or no cardiac imaging. ${ }^{34,35}$ If oncologists are voting with their feet, it appears that recommendations are perceived as promoting overtesting, with a common result being minimal or no testing in actual practice.

\section{A PATH FORWARD}

We suggest a more focused cardiac surveillance approach to low-risk, asymptomatic patients receiving anti-HER2 treatment. Routine serial LVEF measurement by echocardiography should be done only if patients have received anthracyclines or are considered to be at high risk (eg, concomitant hypertension, borderline low LVEF). For these patients, studies should be carried out at baseline, post-anthracycline (if appropriate), and every 3 months while on anti-HER2 treatment. Less frequent testing may be justified for patients with metastatic disease who have repeatedly normal LVEF test results. Patients should be informed about potential symptoms of cardiotoxicity and advised to report them promptly.

\section{DISCLOSURES}

The authors report no relevant financial relationships which, in the context of their contributions, could be perceived as a potential conflict of interest.

\section{REFERENCES}

1. Hudis CA. Trastuzumab-mechanism of action and use in clinical practice. N Engl J Med 2007; 357(1):39-51. doi:10.1056/NEJMra043186

2. Hayes DF. HER2 and breast cancer-a phenomenal success story. $N$ Engl J Med 2019; 381(13):1284-1286. doi:10.1056/NEJMcibr1909386

3. Saini KS, Azim HA Jr, Metzger-Filho O, et al. Beyond trastuzumab: new treatment options for HER2-positive breast cancer. Breast 2011; 20(suppl 3):S20-S27. doi:10.1016/S0960-9776(11)70289-2

4. Ky B, Vejpongsa P, Yeh ET, Force T, Moslehi JJ. Emerging paradigms in cardiomyopathies associated with cancer therapies. Circ Res 2013; 113(6):754-764. doi:10.1161/CIRCRESAHA.113.300218

5. Trastuzumab-Genentech, Inc. Highlights of prescribing information. https://www.accessdata.fda.gov/drugsatfda_docs/ label/2010/103792s5256lbl.pdf. Accessed January 11, 2021.

6. Slamon DJ, Leyland-Jones B, Shak S, et al. Use of chemotherapy plus a monoclonal antibody against HER2 for metastatic breast cancer 
that overexpresses HER2. N Engl J Med 2001; 344(11):783-792. doi:10.1056/NEJM200103153441101

7. Lidbrink E, Chmielowska E, Otremba B, et al. A real-world study of cardiac events in $>3700$ patients with HER2-positive early breast cancer treated with trastuzumab: final analysis of the OHERA study. Breast Cancer Res Treat 2019; 174(1):187-196. doi:10.1007/s10549-018-5058-6

8. von Minckwitz G, Huang CS, Mano MS, et al; for the KATHERINE Investigators. Trastuzumab emtansine for residual invasive HER2positive breast cancer. N Engl J Med 2019; 380(7):617-628. doi:10.1056/NEJMoa 1814017

9. Ganz PA, Romond EH, Cecchini RS, et al. Long-term follow-up of cardiac function and quality of life for patients in NSABP Protocol B-31/NRG Oncology: A randomized trial comparing the safety and efficacy of doxorubicin and cyclophosphamide (AC) followed by paclitaxel with $\mathrm{AC}$ followed by paclitaxel and trastuzumab in patients with node-positive breast cancer with tumors overexpressing human epidermal growth factor receptor 2. J Clin Oncol 2017; 35(35):39423948. doi:10.1200/JCO.2017.74.1165

10. Cameron D, Piccart-Gebhart MJ, Gelber RD, et al; Herceptin Adjuvant (HERA) Trial Study Team. 11 years' follow-up of trastuzumab after adjuvant chemotherapy in HER2-positive early breast cancer: final analysis of the HERceptin Adjuvant (HERA) trial. Lancet 2017; 389(10075):1195-1205. doi:10.1016/S0140-6736(16)32616-2. Erratum in: Lancet 2019; 393(10176):1100. doi: 10.1016/S0140-6736(18)32771-5

11. von Minckwitz G, Procter M, de Azambuja E, et al; for the APHINITY Steering Committee and Investigators. Adjuvant pertuzumab and trastuzumab in early HER2-positive breast cancer. N Engl J Med 2017; 377(2):122-131. doi:10.1056/NEJMoa1703643

12. Dang C, Guo H, Najita J, et al. Cardiac outcomes of patients receiving adjuvant weekly paclitaxel and trastuzumab for node-negative, ERBB2-positive breast cancer. JAMA Oncol 2016; 2(1):29-36. doi:10.1001/jamaoncol.2015.3709

13. Mavroudis D, Saloustros E, Malamos N, et al; Breast Cancer Investigators of Hellenic Oncology Research Group (HORG), Athens, Greece. Six versus 12 months of adjuvant trastuzumab in combination with dose-dense chemotherapy for women with HER2-positive breast cancer: a multicenter randomized study by the Hellenic Oncology Research Group (HORG). Ann Oncol 2015; 26(7):13331340. doi:10.1093/annonc/mdv213. Erratum in: Mavroudis D, Saloustros E, Malamos N, et al; Breast Cancer Investigators of the Hellenic Oncology Research Group (HORG), Athens, Greece. Corrigendum to Six versus 12 months of adjuvant trastuzumab in combination with dose-dense chemotherapy for women with HER2-positive breast cancer: a multicenter randomized study by the Hellenic Oncology Research Group (HORG): Annals of Oncology, Volume 26, Issue 7, July 2015, Pages 1333-1340. Ann Oncol 2020; 31(3):444-445. doi:10.1016/j.annonc.2020.01.004

14. Swain SM, Ewer MS, Cortés J, et al. Cardiac tolerability of pertuzumab plus trastuzumab plus docetaxel in patients with HER2positive metastatic breast cancer in CLEOPATRA: a randomized, double-blind, placebo-controlled phase III study. Oncologist 2013; 18(3):257-264. doi:10.1634/theoncologist.2012-0448

15. Gianni L, Pienkowski T, Im YH, et al. Efficacy and safety of neoadjuvant pertuzumab and trastuzumab in women with locally advanced, inflammatory, or early HER2 positive breast cancer (NeoSphere): a randomised multicentre, open-label, phase 2 trial. Lancet Oncol 2012; 13(1):25-32. doi:10.1016/S1470-2045(11)70336-9

16. Slamon $\mathbf{D}$, Eiermann W, Robert $\mathbf{N}$, et al. Adjuvant trastuzumab in HER2-positive breast cancer. N Engl J Med 2011; 365(14):1273-1283. doi:10.1056/NEJMoa0910383

17. Suter TM, Procter M, van Veldhuisen DJ, et al. Trastuzumab-associated cardiac adverse effects in the herceptin adjuvant trial. J Clin Oncol 2007; 25(25):3859-3865. doi:10.1200/JCO.2006.09.1611

18. Carver JR, Shapiro CL, Ng A, et al. American Society of Clinical Oncology clinical evidence review on the ongoing care of adult cancer survivors: cardiac and pulmonary late effects. J Clin Oncol 2007; 25(25):3991-4008. doi:10.1200/JCO.2007.10.9777

19. Plana JC, Galderisi M, Barac A, et al. Expert consensus for multimo- dality imaging evaluation of adult patients during and after cancer therapy: a report from the American Society of Echocardiography and the European Association of Cardiovascular Imaging. Eur Heart J Cardiovasc Imaging 2014; 15(10):1063-1093. doi:10.1093/ehjci/jeu192

20. Banke A, Fosbøl EL, Ewertz M, et al. Long-term risk of heart failure in breast cancer patients after adjuvant chemotherapy with or without trastuzumab. JACC Heart Fail 2019; 7(3):217-224. doi:10.1016/j.jchf.2018.09.001

21. National Cancer Institute, Division of Cancer Treatment \& Diagnosis. Common Terminology Criteria for Adverse Events (CTCAE). Accessed January 11, 2021. https://ctep.cancer.gov/protocolDevelopment/electronic_applications/ctc.htm\#ctc_50

22. Witteles RM, Fowler MB, Telli ML. Chemotherapy-associated cardiotoxicity: how often does it really occur and how can it be prevented? Heart Fail Clin 2011; 7(3):333-344. doi:10.1016/j.hfc.2011.03.005

23. Witteles RM, Telli M. Underestimating cardiac toxicity in cancer trials: lessons learned? J Clin Oncol 2012; 30(16):1916-1918. doi:10.1200/JCO.2011.40.4012

24. Wilson JM. Multiple screening. Lancet 1963; 2(7298):51-54. doi:10.1016/s0140-6736(63)90059-x

25. Negishi T, Thavendiranathan P, Negishi K, Marwick TH; SUCCOUR investigators. Rationale and design of the Strain Surveillance of Chemotherapy for Improving Cardiovascular Outcomes: the SUCCOUR trial. JACC Cardiovasc Imaging 2018; 11(8):1098-1105. doi:10.1016/j.jcmg.2018.03.019

26. Yu AF, Manrique C, Pun S, et al. Cardiac safety of paclitaxel plus trastuzumab and pertuzumab in patients with HER2-positive metastatic breast cancer. Oncologist 2016; 21(4):418-424. doi:10.1634/theoncologist.2015-0321

27. Nowsheen S, Aziz K, Park JY, et al. Trastuzumab in female breast cancer patients with reduced left ventricular ejection fraction. J Am Heart Assoc 2018; 7(15):e008637. doi:10.1161/JAHA.118.008637

28. Moslehi JJ. Cardiovascular toxic effects of targeted cancer therapies. N Engl J Med 2016; 375(15):1457-1467. doi:10.1056/NEJMra1100265

29. Hussain Y, Drill E, Dang CT, Liu JE, Steingart RM, Yu AF. Cardiac outcomes of trastuzumab therapy in patients with HER2-positive breast cancer and reduced left ventricular ejection fraction. Breast Cancer Res Treat 2019; 175(1):239-246. doi:10.1007/s10549-019-05139-6

30. Koneru S, Sato K, Goldberg A, et al. Pertuzumab/trastuzumab breast cancer therapy is associated with complex hemodynamic abnormalities. JACC Cardiovasc Imaging 2018; 11(8):1206-1207. doi:10.1016/j.jcmg.2018.05.010

31. Koneru S, Collier P, Goldberg A, et al. Temporal variability of global longitudinal strain in stable patients undergoing chemotherapy with trastuzumab. Am J Cardiol 2016; 118(6):930-935. doi:10.1016/j. amjcard.2016.06.030

32. Mescher C, Ding C, Defor T, Konety S, Blaes A. Left ventricular ejection fraction screening and clinical decision-making in metastatic HER2-positive breast cancer. Anticancer Res 2017; 37(7):3751-3755. doi:10.21873/anticanres. 11749

33. Nolan MT, Plana JC, Thavendiranathan P, Shaw L, Si L, Marwick TH. Cost-effectiveness of strain-targeted cardioprotection for prevention of chemotherapy-induced cardiotoxicity. Int J Cardiol 2016; 212:336-345. doi:10.1016/j.ijcard.2016.02.137

34. Chavez-MacGregor M, Niu J, Zhang N, et al. Cardiac monitoring during adjuvant trastuzumab-based chemotherapy among older patients with breast cancer. J Clin Oncol 2015; 33(19):2176-2183. doi:10.1200/JCO.2014.58.9465

35. Pinder MC, Duan Z, Goodwin JS, Hortobagyi GN, Giordano SH. Congestive heart failure in older women treated with adjuvant anthracycline chemotherapy for breast cancer. J Clin Oncol 2007; 25(25):3808-3815. doi:10.1200/JCO.2006.10.4976

Address: Patrick Collier, MD, PhD, FASE, FESC, FACC, Department of Cardiovascular Medicine, Sydell and Arnold Miller Family Heart and Vascular Institute, J1-5, Cleveland Clinic, 9500 Euclid Avenue, Cleveland, $\mathrm{OH}$ 44195; colliep@ccf.org 